\title{
III. Fortbildung und Einsatz der Volksrichter (1946-1952)
}

\author{
1. Rundverfügungen und Berichte zur Fortbildung der Volksrichter
}

\section{Dokument $29^{1}$}

Rundverfügung DJV an Landes- und Provinzialverwaltungen/Abteilung Justiz [gekürzt]; Berlin, November 1946

BAP, DP1 VA Nr.6335, Bl.132-134, Konzept $t^{2}$

Betrifft: Die Fortbildung der Absolventen der Richterlehrgänge

Die Absolventen der Richterlehrgänge bedürfen, wie von ihnen selbst allenthalben anerkannt worden ist, bis auf weiteres noch einer besonderen systematischen Betreuung, da sie während ihrer kurzfristigen Ausbildung nur grundlegende Kenntnisse und Einsichten haben erlangen können. In der Praxis werden sie naturgemäß in erster Linie praktische Fähigkeiten und Kenntnisse vermissen. Hier wird ihnen die beste Hilfe von erfahrenen, akademisch geschulten Richtern und Staatsanwälten gewährt werden; diese Hilfe durch zusammenfassende, allen Absolventen zugute kommende besondere Veranstaltungen zu ergänzen, bleibt eine Aufgabe, die sowohl in den Ländern (Provinzen), vorzugsweise von den Leitern der Lehrgänge, wie zentral von der Deutschen Justizverwaltung in Angriff genommen werden muß. Ich begrüße die an mehreren Stellen bereits geäußerte Absicht der Lehrgangsleiter, mit den Absolventen durch Fernunterricht oder gelegentliche Aussprachen laufend in Gedankenaustausch zu bleiben. Als eine Ergänzung solcher Pläne werden die Schulungsbriefe ${ }^{3}$ wirken, die die Deutsche Justizverwaltung zur Förderung sowohl der ohne juristische Vorbildung im Amte befindlichen Richter und Staatsanwälte wie der Lehrgangsabsolventen zu versenden gedenkt. Diese Briefe werden Fragen, die in der Praxis besonders wichtig sind und die erfahrungsgemäß dem Anfänger oft Schwierigkeiten machen, in einer den Bedürfnissen der Praxis angemessenen knappen Form und in zwangloser Zeit- und Reihenfolge behandeln; für ihren Inhalt tragen nur die Verfasser die Verantwortung. Die Briefe werden durch Fälle, die in der Praxis häufige Themen zum Gegenstand haben, die theoretischen Darlegungen und praktischen Hinweise zu ergänzen und zu beleben suchen. Die Lösung solcher Aufgaben wird regelmäßig in dem folgenden Brief mitgeteilt werden; auch sie ist als persönliche Ansicht der Verfasser zu werten. Diese werden sich für einen im Einzelfall erwünschten Meinungsaustausch zur Verfügung halten und Vorschläge zur Behandlung besonderer Gegenstände gern entgegennehmen; Schreiben solcher Art bitte ich an die Abteilung Ausbildung in der Deutschen Justizverwaltung unmittelbar richten zu lassen.

\footnotetext{
${ }^{1}$ Das Dokument trägt auf der ersten Seite den handschriftlichen Vermerk: „Handexemplar für Frau Vortrag. Rat Dr. Benjamin". Es weist Korrekturen in Wendes und Benjamins Handschrift auf.

${ }^{2}$ Daß das Dokument abgeschickt wurde, geht aus einem Schreiben von Kurt Ebert an den SED-Landesvorstand in Dresden vom 19.12. 1947, BAP, DP1 SE Nr.3561, hervor.

${ }^{3}$ Diese sind mit den sog. Unterrichtsbriefen identisch: siehe dazu Anm. 19 zu Dokument 21.
} 
$[\ldots]^{4}$

Daß Schulungsbriefe niemals eine gutgeleitete mündliche Aussprache oder auch nur einen durch die persönliche Berührung befruchteten mündlichen Vortrag ersetzen können, liegt auf der Hand. Darum bleiben regelmäßige Zusammenkünfte der Lehrgangsabsolventen außerhalb ihres Dienstes während mindestens zwei Jahren nach ihrem Eintritt in die Praxis unerläßlich. In solchen Freizeiten soll einerseits den jungen Richtern und Staatsanwälten Gelegenheit geboten werden, aus ihren Erfahrungen allseitig interessierende Fragen des materiellen und prozessualen Rechts zu einer sachkundig geleiteten und damit ein positives Ergebnis gewährleistenden Aussprache zu bringen; andererseits sollen im Rahmen eines etwa zwei Jahre umfassenden Gesamtplans Aufgaben theoretisch durchgearbeitet werden, deren Behandlung für die Entwicklung des juristischen Könnens der Absolventen von besonderer Bedeutung ist, in dem Lehrgang selbst aber nicht mehr oder noch nicht möglich war. Ziel einer solchen Gemeinschaftsarbeit soll nicht nur die Bearbeitung eines neuen für die Praxis wichtigen Wissensgebietes sein; es soll auch durch Behandlung eines lehrreichen und stofflich bedeutungsvollen Themas die Fähigkeit gestärkt werden, eines in der Praxis auftretenden Problems in selbstverantwortlicher Arbeit Herr zu werden.

Wie die Ergebnisse der Abschlußprüfungen und die ersten Erfahrungen der neuen Kräfte in der Praxis gezeigt haben, besteht das Bedürfnis nach Fortbildung dieser Art bei allen Lehrgangsteilnehmern in gleicher Stärke. Es empfiehlt sich daher, die Aufgaben für die Freizeiten einheitlich für alle Freizeiten auf der Grundlage der Vorschläge der Justizverwaltungen der Länder (Provinzen) zu stellen. Als erste Aufgabe für die mit der Strafrechtspflege befaßten Absolventen wäre voraussichtlich eine Behandlung des gesamten geltenden Wirtschaftsstrafrechts überall willkommen; das Material hierfür würde von der Deutschen Justizverwaltung zur Verfügung gestellt werden können. Für die Zivilrichter erwünscht und nützlich wäre zunächst vielleicht im Rahmen einer Freizeit, die Sonderfragen des Zivilprozesses behandelt, eine Unterweisung im Aufbau eines Zivilurteils; auch hierfür könnte eine Unterlage von hier geliefert werden. Andere Vorschläge der Justizverwaltungen sollen aber auch schon für die erste Freizeit gern erwogen werden.

$[\ldots]^{5}$

\section{Dokument 30}

Bericht über die Sonderveranstaltung zur Fortbildung der Absolventen der Richterlehrgänge, der Referendare und Assessoren in Potsdam am 22. November 1947; Berlin, den 24.11. 1947

$B A P, D P 1$ VA Nr. 10, Bl.80-82, Durchscbrift

Auftragsgemäß haben Frau Direktor Benjamin und der Unterzeichnete an der Sonderveranstaltung zur Fortbildung der Absolventen der Richterlehrgänge, der Referendare und Assessoren in Potsdam am 22. November 1947 teilgenommen.

\footnotetext{
${ }^{4}$ Ausgelassen wurde die Bitte an die Richterschulen und Justizabteilungen der Länder und Provinzen, sich an der Abfassung der Schulungsbriefe zu beteiligen, sowie die Mitteilung, daß die Schulungsbriefe für die Absolventen der Richterschulen und die nicht vorgebildeten Kräfte gedacht seien.

${ }_{5}^{5}$ Ausgelassen wurden Details zu den geplanten Freizeiten.
} 
Die SMAD war durch Oberstleutnant Jakupow vertreten, der bis zum Schluß der Tagung da blieb.

Der Oberlandesgerichtspräsident, der Generalstaatsanwalt und die Landgerichtspräsidenten von Cottbus, Eberswalde und Potsdam nahmen ebenfalls teil. Die Leitung hatte Min[isterial]Dir[ektor] Hoeniger ${ }^{1}$, der sich seiner Aufgabe mit großer Gewandtheit entledigte; gelegentlich zeigte sich, daß er zwar alle Richter des Bezirks genau kennt, aber persönlich nicht immer ein nahes Verhältnis zu ihnen hat. Im ganzen wurden etwa 60 Teilnehmer gezählt, von denen aber nach der späten Mittagspause nur etwa noch die Hälfte wieder erschien. Dies hatte seinen Grund zum Teil in einem Mangel der organisatorischen Vorbereitung, da die meisten Teilnehmer sich darauf eingerichtet hatten, daß die Verhandlungen am 22. November etwa gegen $16 \mathrm{Uhr}$ ihr Ende erreichen sollten. Von der Programmänderung, nach der auch der Sonntag für die Tagung in Aussicht genommen war, hatten sie nichts erfahren, obwohl die neue Tagesordnung bereits am 14. November d.J. in den Händen der Deutschen Justizverwaltung war. Offenbar hatten sich aber nicht nur aus diesen Gründen nicht wenige Teilnehmer vorzeitig entfernt, sondern es wurde auch mehrfach mit Mißfallen festgestellt, daß z.B. Richter und Staatsanwälte, die noch ihren Wohnsitz in Berlin haben, schon in der Mittagspause abgefahren waren. Als ein noch schwererer organisatorischer [Mangel] stellte sich im Laufe der Verhandlungen heraus, daß es bisher - angeblich aus Personalmangel - nicht möglich gewesen ist, eine engere Fühlungnahme zwischen dem Justizministerium und den Absolventen herzustellen, während in den meisten anderen Ländern den Lehrgangsleitern die regelmäßige Betreuung der jungen Richter und Staatsanwälte obliegt, was sich durchaus bewährt hat ${ }^{2}$. Hierauf dürfte es $u . a$. zurückzuführen sein, daß auf die rechtzeitig ergangene schriftliche Aufforderung des Justizministeriums, im voraus Fragen aus der Praxis für die Diskussion anzumelden, nur drei Antworten eingegangen waren. Die Deutsche Justizverwaltung hat schon mehrfach, leider vergeblich, einen engeren Kontakt angeregt; auch hat Frau Direktor Benjamin im Laufe der Verhandlungen nachdrücklich auf diesen Mangel hingewiesen. Die Tagesordnung ist aus der Anlage ersichtlich. Sie wurde im allgemeinen eingehalten mit der Maßgabe, daß es gelang, das Programm schon in den späteren Abendstunden des Sonnabends vollständig abzuwickeln, so daß der Sonntag frei blieb. An Stelle des erkrankten Justizministers begrüßte Min[isterial]dir[ektor] Hoeniger die Erschienenen; er hatte auch an Stelle des dienstlich abberufenen Generalstaatsanwalts Ostmann ${ }^{3}$ das Referat über Zusammenarbeit von Justizverwaltung und Presse übernommen. Für den ebenfalls erkrankten Herrn Fechner vom Zentralsekretariat der SED sprach Herr Schäfermeyer ${ }^{4}$ von der SED über das Thema „Wie schützt der Richter die Demokratie?“. Oberstleutnant Jakupow machte einleitend längere Ausführungen über die Demokratisierung der Justiz. Über die Aufgaben der jungen Richter und Staatsanwälte und über die Notwendigkeit der strengen Bestrafung von Wirtschaftsvergehen, namentlich bei Nichterfüllung des Abgabesolls, wobei er einzelne Urteile aus dem Cottbuser Bezirk kritisch anführte. (Hierzu gab demnächst in der Diskussion St[aats]A[nwalt]

\footnotetext{
1 Walter Hoeniger (SPD/SED), 1946-1948 Leiter der Abteilung Justiz in der Provinzialverwaltung Brandenburg bzw. im Justizministerium Brandenburg.

2 Siehe Einleitung, S. $72 \mathrm{f}$.

${ }^{3}$ Zu Ostmann siehe Anm. 11 zu Dokument 14.

${ }^{4}$ Reinhold Schäfermeyer (SED), stellvertretender Leiter der Abteilung Justiz beim SED-Zentralsekretariat.
} 
Wiederski ${ }^{5}$ vom Landgericht in Cottbus einige richtigstellende Erklärungen ab.) Der Unterzeichnete überbrachte die Grüße des Amtschefs der DJV. Er ging im Anschluß daran insbesondere auf die Versuchungen ein, denen die jungen Richter und Staatsanwälte ausgesetzt sind (Schwatzhaftigkeit, Schwarzer Markt, Bestechung).

Die gehaltenen Referate litten zum Teil etwas darunter, daß sie zu allgemein gehalten waren und den Bedürfnissen der Praxis nicht genügend Rechnung trugen. Das hob in der Debatte auch Oberstleutnant Jakupow hervor; er wandte sich des weiteren im Anschluß an das Referat von Min[isterial]Dir[ektor] Hoeniger über Bewährungseinsatz gegen die Neigung, den Strafvollzug allzu human zu gestalten ${ }^{6}$ : Es gehe nicht an, die Verurteilten im Bewährungseinsatz den freien Arbeitern völlig gleichzustellen und sie die Strafe als solche nicht spüren zu lassen. Auch schien es ihm zu weit zu gehen, wenn ein Monat Bewährungseinsatz auf die Strafe mit zwei Monaten angerechnet werde; wenn die Notwendigkeit des Bewährungseinsatzes zum Teil mit der Überfüllung der Gefängnisse begründet werde, so müsse man sich vor Augen halten, daß diese Überfüllung vor allem durch die allzu lange Dauer der Untersuchungshaft verursacht werde, zumal die Untersuchungsgefangenen etwa $75 \%$ aller Häftlinge stellten.

In der Diskussion fielen mehrere junge Richter und Staatsanwälte durch ihr gewandtes und sachlich begründetes Vorbringen angenehm auf; an erster Stelle ist hier der schon erwähnte Staatsanwalt Wiederski in Cottbus zu nennen.

gez. Dr. Hartwig

\section{Dokument 31}

Rundverfügung Chef der DJV an Landesregierungen/Justizministerium; Berlin, den 29.7. 1948

BAP, DP1 VA Nr. 1041, Durchschrift

Betrifft: Weiterbildung der Absolventen der Lehrgänge für Richter und Staatsanwälte

Eine der wichtigsten Aufgaben der Justizverwaltung ist die Weiterbildung der Absolventen der Lehrgänge für Richter und Staatsanwälte. Wenn auch hier bereits manche Erfolge erzielt worden sind, so werden doch alle Beteiligten, einschließlich der Absolventen selbst, sich darüber einig sein, daß noch mehr geschehen muß, um das berufliche Können aller Absolventen soweit zu fördern, daß sie ihren Platz voll ausfüllen.

Im folgenden sollen deshalb die wesentlichsten Gesichtspunkte, die dabei zu beachten sein werden, kurz zusammengefaßt werden:

1. Die Absolventen müssen nach Möglichkeit solchen Gerichten und Staatsanwaltschaften zugeteilt werden, bei denen nach den Geschäftsverhältnissen und Persönlichkeiten der

${ }^{5}$ Gemeint war Staatsanwalt Helmut Wensierski aus Cottbus: siehe den Bericht von Götz Berger über dieselbe Veranstaltung, BAP, DP1 VA Nr.6596, Bl.12-17.

${ }^{6} \mathrm{Zu}$ den Reformbestrebungen im Strafvollzug, die auch von dem Leiter der zuständigen Abteilung in der DJV, Werner Gentz, verfolgt wurden, siehe Oleschinski, Abteilung Strafvollzug. Auch Hoeniger zählte zu den Befürwortern eines humaneren Strafvollzugs: siehe dazu Hoeniger, Bewährungseinsatz, S. 178-180. 
übrigen dort vorhandenen Richter und Staatsanwälte die Gewähr für ihre sachgemäße Weiterbildung gegeben erscheint. Schon aus diesem Grunde empfiehlt sich im Anfange nicht ihre Verwendung in Einzelrichterstellen, wo sie ganz auf sich gestellt und gezwungen sind, sich gleichzeitig mit den verschiedensten Rechtsgebieten zu befassen. Die Absolventen dürfen ihre Dienststelle und ihre Tätigkeit nicht zu häufig wechseln, damit sie sich in Ruhe einarbeiten und mit den örtlichen Verhältnissen, auch außerhalb der Justiz, genügend vertraut machen können. Es ist darauf Bedacht zu nehmen, daß sie nur solchen Kammervorsitzenden zugeteilt werden, die es als ihre selbstverständliche Pflicht betrachten, die Beisitzer auf jede mögliche Weise zu fördern, die sie ausreichend zu Wort kommen lassen, die alle gefertigten Urteile und Beschlüsse mit ihnen besprechen und sie in angemessener Form auf die gemachten Fehler aufmerksam machen. Die Vorsitzenden müssen sich bewußt bleiben, daß sie den Absolventen einen schlechten Dienst leisten, wenn sie - wie es gelegentlich vorgekommen ist - „der Einfachheit halber“ Urteile und Beschlüsse selbst absetzen. Möglichst frühzeitig sind die Absolventen mit der Verhandlungsleitung und mit der Wahrnehmung des Sitzungsdienstes der Staatsanwaltschaft zu betrauen. Eine baldige feste Anstellung und zu gegebener Zeit - im Falle allseitiger Bewährung - eine Beförderung wird den Eifer und die Leistungen der Absolventen heben.

2. Die allgemeine Leitung der Weiterbildung der Absolventen ist in eine Hand zu legen. Es erscheint zweckmäßig, hiermit den Lehrgangsleiter zu beauftragen, dem die Absolventen zum großen Teil bekannt sind und bei dem das erforderliche Verständnis, pädagogische Erfahrung und Interesse vorausgesetzt werden können. Er wird dafür Sorge tragen müssen, daß die Absolventen regelmäßig und pünktlich die von der Deutschen Justizverwaltung herausgegebenen Unterrichtsbriefe ${ }^{1}$ erhalten, und sich bei jeder sich bietenden Gelegenheit davon zu überzeugen haben, daß sich die Absolventen den Inhalt dieser Briefe zu eigen gemacht haben. Ferner wird er die ständige Verbindung mit jedem einzelnen Absolventen aufrechterhalten müssen. Diese werden ihm etwa alle 2 Monate schriftlich über die in der $Z$ wischenzeit von ihnen geleistete Arbeit, auch außerhalb der eigentlichen Berufsaufgaben und die dabei aufgetauchten Zweifelsfragen zu berichten und Vorschläge über die weitere Ausgestaltung der Lehrgänge zu unterbreiten haben. Von Zeit zu Zeit wird der Lehrgangsleiter an den zu 3 zu erwähnenden Zusammenkünften der Absolventen am Sitz der Landgerichte teilzunehmen haben.

3. Die Absolventen eines jeden Landgerichtsbezirks sind zu einer Gruppe zusammenzufassen. Diese Gruppe ist unter die Leitung eines nach seiner Persönlichkeit, seiner fortschrittlichen Einstellung und seinem Können besonders dafür befähigten Richters oder Staatsanwalts zu stellen. Es darf erwartet werden, daß viele Landgerichtspräsidenten und Oberstaatsanwälte sich im Hinblick auf die Wichtigkeit der lohnenden Aufgabe bereit erklären, selbst die Leitung zu übernehmen. Die persönliche Fühlungnahme und Einwirkung steht hier im Vordergrund. Sie darf sich selbstverständlich nicht auf die am Sitze des Landgerichts selbst befindlichen Angehörigen der Gruppe beschränken. Etwa einmal im Monat hat der Leiter die ganze Gruppe für mehrere Stunden um sich zu versammeln und mit ihr nicht nur neue Gesetze und Verordnungen und sonstige wichtige Fragen der Praxis zu besprechen, sondern auch ihre Berufsauffassung zu festigen, den fortschrittlichen Geist wach zu halten und sie immer wieder darauf hinzuweisen, daß die Arbeit nicht mit der Erledigung der vorliegenden Akten beendet ist, sondern daß die Absolventen sich am öffentlichen Leben weitgehend beteiligen und enge Fühlung mit den übrigen

\footnotetext{
${ }^{1}$ Siehe Anm. 19 zu Dokument 21.
} 
Behörden, den politischen Parteien und den demokratischen Organisationen sowie dem wirtschaftlichen Leben, der Presse und den sozialen und kulturellen Gerichtseingesessenen halten müssen. Auch wird der Leiter ihnen Fingerzeige für ihr weiteres Privatstudium unter Namhaftmachung der hierfür in Betracht kommenden Literatur zu geben haben. Seine Tür muß allen von ihm Betreuten jederzeit offenstehen. Etwa alle 3 Monate wird er dem Lehrgangsleiter von seinen Eindrücken unter Beifügung einer eingehenden Beurteilung eines jeden einzelnen Mitteilung zu machen haben.

4. Mehrmals im Jahre sind alle Absolventen aus dem ganzen Oberlandesgerichtsbezirk für kürzere oder längere Zeit zu einer besonderen Fortbildungstagung zusammenzufassen. Das Gelingen einer solchen Veranstaltung hängt ganz wesentlich von einer sorgfältigen Vorbereitung ab. Insbesondere wird dafür Sorge zu tragen sein, daß wichtige und gegenwartsnahe Fragen zum Gegenstande der Vorträge und Diskussionen gemacht werden und daß mit den Vorträgen, die sich keineswegs auf rein juristische Fragen zu beschränken brauchen, besonders geeignete, kenntnisreiche und lebendige Persönlichkeiten betraut werden. Oft wird es sich auch empfehlen, einzelne Diskussionsredner schon im voraus zu bestimmen. Der Zeitpunkt und das Programm der Tagung wird allen Teilnehmern geraume Zeit vorher mitzuteilen sein, damit sie bei der Anberaumung von Sitzungen und Terminen darauf Rücksicht nehmen und sich mit der Tagungsordnung vertraut machen können.

5. Bei allen Geschäftsrevisionen muß der Besichtigende sich mit der Tätigkeit, der Leistung und der gesamten Persönlichkeit des einzelnen Absolventen befassen; er hat dafür Sorge zu tragen, daß seine Feststellungen und Beurteilungen an die maßgebenden Stellen gelangen.

6. Alles vorstehend Gesagte gilt nicht nur für die Absolventen, sondern in gleicher Weise auch für die Richter und Staatsanwälte ohne besondere Vorbildung.

Ich bitte, zum 1.Dezember 1948 über das Veranlaßte und die gemachten Erfahrungen zu berichten.

Schiffer

\section{Dokument 32}

Bericht über die Fortbildungsveranstaltung der Absolventen des 1. und 2. Richter-Lehr gangs in Bad Schandau vom 18.-21. August 1948; Berlin, den 1.9. 1948

$B A P, D P 1$ VA Nr.6335, Durchschrift

Von insgesamt 48 im Lande Sachsen tätigen Absolventen des 1. und 2. Richter-Lehrgangs haben 34 an der Veranstaltung teilgenommen, 12 fehlten entschuldigt und 2 unentschuldigt. Die Veranstaltung trug durchaus zwanglosen Charakter. Der erste Tag war den Berichten der Teilnehmer gewidmet. Am zweiten Tag sprach vormittags Generalstaatsanwalt Helm ${ }^{1}$ über die allgemeine Lage in der Justiz und über Probleme des Wirtschaftsstrafrechts und der 201-Verfahren ${ }^{2}$. Der Nachmittag brachte ein Referat des Min[isterial]Rats

\footnotetext{
${ }^{1}$ Zu Helm siehe Anm.11 zu Dokument 23.

2 Siehe dazu Einleitung, S. 87.
} 
$\mathrm{Cohn}^{3}$ über die Fragen der Strafzumessung. Am 20.8., vormittags, referierte Min[isterial] Direktor Dr. Mannsfeld ${ }^{4}$ über Fragen des Zivilprozesses; am Nachmittag leitete Präsident Dr. Ebert eine Aussprache über Fragen des Zivilrechts. Der Vormittag des letzten Tages, am 21.8., war für ein Referat des A[mts]g[erichts]rats Fabisch über Fragen der Zwangsvollstreckung vorbehalten.

Es war sehr von Vorteil, daß die Referate nur höchstens 11/2 bis 2 Stunden dauerten, so $\mathrm{da}$ noch ausreichende Zeit für die Diskussion zur Verfügung stand. Das Ergebnis der Diskussion führte zu folgender grundsätzlichen Feststellung.

Für diese Richter, die jetzt ein bis zwei Jahre in der Praxis stehen, gibt es keine besonderen „Volksrichter-Probleme“ mehr. Die Fragen, die sie behandelten, sind allgemeine Justizprobleme. Es sind die Probleme des fortschrittlichen Richters gegenüber rückschrittlichen Strömungen in der Justiz, und es sind die Probleme der Praxis, die vor jedem Richter stehen.

An der Diskussion beteiligte sich etwa die Hälfte der Teilnehmer, wobei sich ergab, daß der Kreis dieser aktiven Richter und Staatsanwälte bei sämtlichen Themen im allgemeinen der gleiche war.

Zur Charakteristik für die Vielseitigkeit der Aussprache seien einige Diskussionspunkte genannt:

Die besondere Lage im erzgebirgischen Bergbaugebiet (Schneeberg, Aue) ${ }^{5}$ in bezug auf Kriminalität, Vollstreckung der Strafen (Probleme des Bewährungseinsatzes im Bergbau), das Verhalten der Justizangestellten (Abwanderung zu den Bergbaubetrieben wegen der besseren Bezahlung und Versorgung), Fragen des Strafvollzugs, Bewährungseinsatz, Bewährungshaftlager, Fürsorge für entlassene Strafgefangene; Einführung einer besonderen Bagatell-Gerichtsbarkeit; die Frage der Anwaltshonorare besonders in Wirtschaftssachen (wird nicht der Anwalt zum Helfer?); Fragen aus dem Zivilrecht; Zwangsvollstreckung nach $\$ 825 \mathrm{ZPO}^{6}$; Sowjet-A.G.? als Drittschuldner; praktische Fragen bezüglich der Auswirkung der Währungsreform ${ }^{8}$. Zu dem Thema „Strafzumessung“ kam es zu einer besonderen Diskussion auf der Grundlage der in der „Neuen Justiz“ angeschnittenen Frage: "Leugnen als Strafzumessungsgrund“9. Diese Themen stellen einen kleinen Teil der mit großer Verantwortung erörterten wichtigen Fragen dar.

${ }^{3}$ Kurt Cohn (LDP), Ministerialrat im Mdj Sachsen 1947-1949; zu seiner weiteren Karriere siehe Rottleuthner, Steuerung, S. 540.

${ }^{4}$ Ernst Mannsfeld (LDP), Leiter der Hauptabteilung Öffentliches Recht und Sozialrecht, bürgerliche Rechtspflege, Personal- und Wirtschaftsverwaltung im MdJ Sachsen.

${ }^{5}$ Im Erzgebirge wurde in sowjetischer Regie seit Ende 1945 Uran abgebaut. Auf Beschluß des Ministerrats der UdSSR vom Mai 1947 erfolgte die Gründung der Sowjetischen Aktiengesellschaft "Wismut", die fortan für den Uranabbau verantwortlich war: siehe dazu zuletzt Karlsch, Aufbau der Uranindustrie.

6 $\$ 825$ ZPO lautet: „Auf Antrag des Gläubigers oder des Schuldners kann das Vollstreckungsgericht anordnen, daß die Verwertung einer gepfändeten $\mathrm{Sache}$ in anderer Weise oder an einem anderen Ort, als in den vorstehenden Paragraphen bestimmt ist, stattzufinden habe oder daß die Versteigerung durch eine andere Person als den Gerichtsvollzieher vorzunehmen sei."

7 Sowjetische Aktiengesellschaften (SAG) waren in der SBZ gegründete sowjetische Staatskonzerne, denen die nach SMAD-Befehl Nr.167 vom 5.6. 1946 beschlagnahmten deutschen Industriebetriebe angegliedert wurden.

${ }^{8}$ Nachdem am 20.6. 1948 in den Westzonen eine Währungsreform durchgeführt worden war, folgte die SBZ mit ihrer eigenen Wahrungsreform am 21.6. 1948.

9 Unter dem Titel "Geständnis und Leugnen als Strafzumessungsgründe ${ }^{\alpha}$ war eine Zuschrift in der NJ abgedruckt worden, die eine Diskussion unter den Lesern auslöste. Diese ist unter dem Titel 
Für die künftige Tätigkeit der Absolventen der Lehrgänge sind vor allem noch zwei Gesichtspunkte, die unter allgemeiner Zustimmung hervorgehoben wurden, von Bedeutung. Es wurde darüber Klage geführt, daß die insbesondere von den Absolventen der Lehrgänge in der Erstinstanz gefällten fortschrittlichen Urteile durch die überwiegend von überalterten Richtern besetzten Berufungskammern häufig abgeändert werden und damit die Tätigkeit der fortschrittlichen Kräfte durch die Berufungsinstanz illusorisch gemacht wird. Weiter wurde angeregt, daß die Richter und Staatsanwälte, die nun schon seit einem Jahr 201-Sachen bearbeiten, im Interesse der Vielseitigkeit ihrer Ausbildung wieder in die allgemeine Praxis zurückgehen und durch neue Kräfte zum mindesten allmählich abgelöst werden. Da Generalstaatsanwalt Dr. Helm damit rechnet, daß die 201-Sachen noch bis in das Jahr 1950 hineinlaufen werden, erscheint diese Anregung sehr wichtig.

Als Folgerung aus der Beobachtung der Entwicklung dieser Richter, die besonders deutlich bestätigt, was bereits auf der zwei Wochen früher besuchten Fortbildungsveranstaltung in Finsterbergen ${ }^{10}$ erkennbar war, ergibt sich:

1.) Die Fortbildungsveranstaltungen für die Absolventen der Richterlehrgänge sind in Zukunft zu differenzieren. Für die Absolventen des 1. und 2. Lehrgangs, deren praktisches Wissen anerkennenswert vorgeschritten ist, sind besondere Veranstaltungen nicht mehr erforderlich. Es sind vielmehr in gleicher Art, wie bisher die Fortbildungsveranstaltungen für die Absolventen der Lehrgänge durchgeführt wurden, Veranstaltungen abzuhalten, in denen neben den Absolventen überhaupt jüngere und entwicklungsfähige Richter und Staatsanwälte, gegebenenfalls auch schon Referendare des 3. Ausbildungsjahres, zusammengefaßt werden. Auf diesen Veranstaltungen sind sowohl Fragen der Praxis, wie allgemeine politische und justizpolitische Fragen zu behandeln. Die Gruppe der SEDTeilnehmer des Lehrgangs in Finsterbergen gibt insofern eine gute Anregung.

2.) Die neu in die Praxis eintretenden Richter und Staatsanwälte sind zu besonderen Lehrgängen, in denen insbesondere auch ihre juristischen und praktischen Kenntnisse ergänzt werden, zusammenzufassen. Zu diesen Lehrgängen sollen auch Absolventen der früheren Lehrgänge, soweit sie der Praxis noch nicht voll genügen, hinzugezogen werden.

3.) Die Absolventen des 1. und 2. Lehrgangs sind nunmehr, wie das von der Unterzeichneten anläßlich der Juristenkonferenz am 11. und 12. Juni 1948 bereits angeregt wurde, auch als Vorsitzende erstinstanzlicher Kammern und von Berufungskammern sowie als Leiter von Amtsgerichten zu verwenden ${ }^{11}$.

4.) Die Richter, die bisher ein Jahr ausschließlich oder überwiegend mit der Bearbeitung von 201-Sachen beschäftigt wurden, sind abzulösen und in die allgemeine Praxis zu überführen.

Berlin, den 1.9. 1948

gez. Benjamin.

${ }_{\text {nGeständnis und Leugnen als Strafzumessungsgründe }}{ }^{\alpha}$ in einem späteren Heft dokumentiert: siehe NJ 2 (1948), S. 48, 108-110.

10 Siehe Bericht über die Teilnahme an der Fortbildungsveranstaltung der Absolventen der Lehrgänge in Finsterbergen vom 2.-14.8. 1948, BAP, DP1 VA Nr.6335.

"Siehe dazu Einleitung, S.89, und Dokument 39. 


\section{Dokument 33}

Fernschreiben Chef der DJV an Landesregierungen/Justizministerium; Berlin, den 24.12. 1948

BAP, DP1 VA Nr.7844, Bl. 299, Durchschrift

Um die mit der Arbeit von Wirtschaftsstrafsachen befaßten Richter und Staatsanwälte mit ihren Aufgabengebieten schnell näher vertraut zu machen, ist die Einrichtung von besonderen Fortbildungsveranstaltungen notwendig. Diese Lehrgänge sollen möglichst noch im Monat Januar stattfinden. Sie sind auf die Dauer von 2 Wochen berechnet und werden internatsmäßig abgehalten. Soweit der Justizverwaltung keine geeigneten Räume zur Verfügung stehen, empfiehlt es sich, sich um die Überlassung von Verwaltungs- und FDGBSchulen zu bemühen. Ein Lehrgang soll etwa 30 Teilnehmer umfassen. Ist der Kreis der zur Teilnahme in Frage kommenden Richter größer, muß eine Wiederholung stattfinden.

Der Teilnehmerkreis umfaßt:

1.) den Wirtschaftsstaatsanwalt beim Generalstaatsanwalt,

2.) die Wirtschaftsstaatsanwälte bei den Oberstaatsanwälten,

3.) 1 Richter des Oberlandesgerichts,

4.) a) bei Mecklenburg, Brandenburg, Thüringen und Sachsen-Anhalt: je 1 Richter jedes Landgerichts (den Vorsitzenden einer Strafkammer),

b) bei Sachsen je 2 Richter der Landgerichte Leipzig, Dresden, Chemnitz und Zwikkau sowie je 1 Richter der anderen Landgerichte (den Vorsitzenden einer Strafkammer),

5.) Richter der Amtsgerichte, wobei die Amtsgerichte, bei denen Wirtschaftsstrafsachen besonderer Wichtigkeit vorkommen, zuerst zu berücksichtigen sind. Die Liste der Teilnehmer ist der Deutschen Justizverwaltung bis zum 5.1. 1949 zur Bestätigung vorzulegen.

Als Leiter des Lehrganges wird empfohlen, den Referenten des Justizministeriums, der mit der Kontrolle der Rechtsprechung in Wirtschaftsstrafsachen befaßt ist, zu bestellen.

Das Lehrprogramm soll folgende Punkte umfassen:

I. Wirtschaftliche und politische Themen
a) die Wirtschaft der Ostzone,
b) der Zweijahresplan,
c) die volkseigenen Betriebe,
d) Planung und Produktion,
e) Verteilung,
f) die landwirtschaftliche Planung.

II. Juristische Themen:
a) Wirtschaftsstrafverordnung ${ }^{1}$,
b) sonstige wirtschaftsstrafrechtliche Bestimmungen,
c) die besonderen Aufgaben der Staatsanwaltschaft in Wirtschaftsstrafverfahren,

1 Verordnung über die Bestrafung von Verstößen gegen die Wirtschaftsordnung, 23.9. 1948, in: Zentralverordnungsblatt 1948, S. 439-443. 
d) Zusammenarbeit von Justiz und Verwaltung,

e) Abfassung von Anklageschriften und Urteilen in Wirtschaftsstrafsachen,

f) die richtige Strafzumessung.

Für diese Themen sind insgesamt 44 Vorlesungsstunden vorgesehen, die durch Seminar, Selbststudium und Betriebsbesichtigungen zu ergänzen sind. Als weitere Themen kommen noch Vernehmungstechnik und Zusammenarbeit mit der Presse in Frage. Die Deutsche Justizverwaltung wird bis zum 5.1. 1949 den Justizministerien die genaue Stundenverteilung sowie ausführliche Dispositionen für die Referate zur Verfügung stellen ${ }^{2}$. Ich bitte bereits jetzt, geeignete Lehrkräfte für dieses Thema auszuwählen und mir ebenfalls bis zum 5.1. 1949 namhaft zu machen.

Für Anfang Januar ist eine Arbeitsbesprechung der Lehrgangsleiter bei der Deutschen Justizverwaltung vorgesehen. Ich bitte, die Vorbereitungen für diesen außerordentlich wichtigen Lehrgang sofort in Angriff zu nehmen.

In Vertretung

Dr. Melsheimer

\section{Dokument 34}

Rundverfügung Nr.101/50, MdJ an Landesregierungen/Justizministerium; Berlin, den 17.8. 1950

\section{BAP, DP1 SE Nr.3548, Durchschrift}

Betr.: Monatliche Fortbildungsveranstaltungen für Richter und Staatsanwälte Bezug: Rundschreiben der Deutschen Justizverwaltung vom 26.9. 1949 - 2210 b - R I 2 $630 / 49-1$

Bis zum Beginn einer systematischen Fernschulung der in der Praxis tätigen Richter und Staatsanwälte stellt die monatliche Fortbildungsveranstaltung im Landgerichtsmaßstab die einzige Möglichkeit der Justizverwaltung dar, das weltanschauliche und gleichzeitig das fachliche Wissen aller leitenden Angestellten der Justiz entscheidend zu verbessern. Die Feststellungen des Justizministeriums der Deutschen Demokratischen Republik haben ergeben, daß vielerorts die Bedeutung der Fortbildungstagungen weder von den Veranstaltern, noch von den Teilnehmern erkannt worden ist. Dies zeigt sich schon rein äußerlich darin, daß die Tagung auf wenige Stunden zusammengedrängt wird, um den Teilnehmern die Unbequemlichkeit des frühen Aufstehens und des späten Nachhausekommens zu ersparen. Dies ergibt sich auch aus den viel zu geringen Teilnehmerzahlen, die darauf schließen lassen, daß die Anwesenheit der einzelnen Tagungsteilnehmer nicht namentlich festgestellt wird oder daß sich die Veranstaltungsleitung mit formalen Entschul-

2 Siehe dazu Chef der DJV an Landesregierungen/Justizministerium, 6.1. 1949, BAP, DP1 VA Nr.7844, Bl. 300. Mit dieser Rundverfügung wurden freilich nur Dispositionen zu den juristischen Themen übersandt; die DWK hatte bis zu diesem Zeitpunkt der DJV nur zu dem Thema "Planung der Verteilung " eine Disposition zukommen lassen.

1 Chef der DJV an Landesregierungen/Justizministerium, 26.9. 1949, BAP, DP1 VA Nr.7094. Der Teil der Rundverfügung zur Fortbildung der Lehrgangsabsolventen ist in Dokument 21 zitiert. 
digungen begnügt. Dies zeigt sich aber auch an der vielfach nachlässigen und sich in allgemeinen Redensarten ergehenden Berichterstattung, die von den Justizministerien der Länder geduldet wird. Dies ist schließlich zu ersehen aus der negativen Einstellung vieler Richter und Staatsanwälte selbst, die die Fortbildungsveranstaltung als „Zeitversäumnis“ betrachten, die sie von ihrer „eigentlichen“ Arbeit „abhält". Diese Einstellung steht in schärfstem Gegensatz zu dem immer wieder an mich herangetragenen Wunsche der Richter und Staatsanwälte, ihnen Möglichkeiten der Fortbildung zu eröffnen. Ein Landgerichtspräsident, der angesichts der vielen drängenden Gegenwartsfragen, die die Justiz der Deutschen Demokratischen Republik betreffen, nicht in der Lage ist, monatlich eine Fortbildungsveranstaltung zu leiten, die von allen Teilnehmern als unbedingt notwendig und der Verbesserung ihrer praktischen Arbeit dienlich anerkannt wird, erfüllt seine Aufgabe schlecht.

Im folgenden wird eine Reihe von konkreten Vorschlägen angeführt, die ich im Interesse der Verbesserung der Schulungsarbeit genau zu beachten bitte.

\section{1.) Organisation}

Die Fortbildungsveranstaltungen müssen zu einem festen Bestandteil der Justizschulung werden. Dazu ist es notwendig, sie in jedem Monat für jeden Landgerichtsbezirk an bestimmten, feststehenden Tagen durchzuführen. Auf diese Weise wird von den Justizbehörden von vornherein dieser Tag terminfrei zu halten sein, so daß es weder der wochenlang vorherigen Ankündigung noch der Umdisponierung von Terminen bedarf.

Selbstverständlich muß die Teilnahme an der Fortbildungsveranstaltung für alle Richter, Staatsanwälte und von den jeweiligen Justizministerien zu bestimmende weitere Justizangestellte Dienst und darum Pflicht sein. Außerdem sollte unbedingt ein Vertreter der höheren Justizorgane (Justizministerien, Oberlandesgerichte oder Generalstaatsanwaltschaften) in jeder Fortbildungsveranstaltung anwesend sein.

2.) Auswahl der Themen

Die Themen für die Fortbildungsveranstaltungen müssen von der Abteilung Schulung in engster Zusammenarbeit mit der Abteilung Rechtsprechung des Landesjustizminsteri$u^{2} s^{2}$ aufgestellt werden und in allen Landgerichtsbezirken monatlich die gleichen sein. Dabei ist, da die Fortbildungsveranstaltung sowohl der ideologischen als auch der fachlichen Vervollkommnung dienen soll, jeweils ein politisches oder wirtschaftspolitisches Thema und ein fachliches Thema zu empfehlen. Das Ministerium der Justiz der Deutschen Demokratischen Republik wird sich zunächst noch darauf beschränken, lediglich Anregungen für die Themenstellung zu geben, wie es beispielsweise in letzter Zeit durch die Rundverfügung vom 24.6. $1950 \mathrm{Nr} .80 / 50^{3}$ geschehen ist. Im übrigen werden neben rein fachlich-theoretischen Themen auch grundsätzliche Fehler formeller und materieller Art, wie sie von den Abteilungen Rechtsprechung der Ministerien der Länder festgestellt

\footnotetext{
${ }^{2}$ Nach der Umstrukturierung der Landesjustizministerien Anfang 1949 verfügten diese einheitlich über die Abteilungen Personal und Schulung sowie Kontrolle und Anleitung der Gerichte: siehe Anders, Demokratisierung, S. 202.

${ }^{3}$ Rundverfügung Nr.80/50 vom 24.6. 1950, BAP, DP1 VA Nr.5576, bezog sich auf den sog. „Dessauer Prozeß" ${ }^{\prime}$, einen der ersten großen Schauprozesse. Bei diesem gegen die Deutsche-Continental-Gas A.G. gerichteten Verfahren waren Leo Herwegen, Georg Brundert und andere zu mehrjährigen Zuchthausstrafen verurteilt worden. In der Rundverfügung erging die Anweisung, den Prozeß "bei den Arbeitsbesprechungen in allen Justizorganen, auf Richtertagungen und in Justizausspracheabenden zum Gegenstand der Erörterungen durch Referate und Diskussionen" zu machen.
} 
werden, zu behandeln sein, wie auch der Inhalt der Halbjahresberichte, die Ergebnisse der statistischen Feststellungen und die sich jeweils ergebenden Schwerpunktaufgaben.

3.) Referenten

Große Sorgfalt ist auf die Auswahl der Referenten zu legen. Es sollten nur Referenten bestellt werden, von denen feststeht, daß sie den Teilnehmern etwas zu geben imstande sind. Es ist unter Umständen produktiver und erfolgversprechender, einen Referenten vom Justizministerium auszuwählen, der sein Thema in allen Landgerichtsbezirken zu halten hat, als die Auswahl der Referenten den einzelnen Landgerichtsbezirken zu überlassen. Bei der Behandlung rein fachlicher Themen wird grundsätzlich auf sachkundige und geschulte Justizangehörige zurückzugreifen sein, während für das politische oder wirtschaftspolitische Thema geeignete Referenten auch aus anderen Verwaltungsstellen und Massenorganisationen gewonnen werden sollten.

4.) Vorbereitung

Bei entsprechender Organisation (siehe 1) genügt es, etwa 3 Wochen vorher den Justizbehörden des betreffenden Landgerichtsbezirks von den Themen und Referenten für die nächste Fortbildungsveranstaltung Mitteilung zu machen. Es empfiehlt sich, gleichzeitig ein Quellen- und Literaturverzeichnis über das zu behandelnde Thema aufzustellen, damit allen Teilnehmern an der Schulungsveranstaltung die Möglichkeit gegeben ist, sich entsprechend darauf vorzubereiten.

\section{5.) Protokolle}

Über jede Veranstaltung ist ein Protokoll anzufertigen, in dem nicht eine umfangreiche Wiedergabe des Verhandlungsablaufes niedergelegt, sondern eine konzentrierte und sinnvolle Darstellung des behandelten Stoffes und der Diskussion gegeben werden soll. Hierbei sind zweckmäßig als Protokollführer solche Justizangestellte heranzuziehen, die gewöhnlich die Sitzungsprotokolle in Strafsachen führen. Von diesem Protokoll bitte ich unverzüglich Abschriften dem Ministerium der Justiz der Deutschen Demokratischen Republik zu übersenden.

\section{6.) Voranzeigen}

Ich bitte, dem Ministerium der Justiz der Deutschen Demokratischen Republik rechtzeitig über die in den Landgerichtsbezirken angesetzten Veranstaltungen Kenntnis zu geben, um die Teilnahme gegebenenfalls auch Mitarbeitern meines Ministeriums zu ermöglichen. Die Bekanntgabe der Termine wird zweckmäßigerweise mit der künftigen Monatsberichterstattung an die Abteilung Schulung (hierüber ergehen noch Anweisungen) zu verbinden sein. Außerdem empfehle ich, der Schulungsabteilung des zuständigen Innenministeriums von diesen Veranstaltungen mit Themenplan und Referentenangabe Mitteilung zu machen.

Fechner 


\section{Dokument 35}

Bericht über die gemeinsamen Länderkonferenzen der Generalstaatsanwaltschaften mit den Landesjustizverwaltungen über das Thema: „Gesetz zum Schutze des innerdeutschen Handels“; Berlin, den 31.8. $1951^{1}$

\section{$B A P, D P 1 S E$ Nr.3548, Original}

Die Länderkonferenzen sind vom 20. bis 24. August 1951 in allen Ländern der Deutschen Demokratischen Republik durchgeführt worden.

Teilgenommen haben die Mitarbeiter der Generalstaatsanwälte und der Justizverwaltungen der Länder, die mit Wirtschaftsstrafsachen befaßten Richter und Staatsanwälte des Landes, die Oberlandesgerichtspräsidenten, Landgerichtspräsidenten und Oberstaatsanwälte.

Es referierte in allen Konferenzen Staatsanwalt Grube ${ }^{2}$ vom Generalstaatsanwalt der Deutschen Demokratischen Republik.

Im Vordergrund der Diskussion stand, wie es meistens der Fall ist, die Behandlung von Fragen und Unklarheiten, die sich für die Richter und Staatsanwälte aus den ihnen zur Zeit vorliegenden Verfahren ergeben.

\section{Allgemeine Eindrücke}

Besucht wurden von mir die Veranstaltungen in Dresden, Erfurt und Halle.

a) Bei der Differenzierung der Straftaten, die unter das Gesetz zum Schutze des innerdeutschen Handels fallen, besteht bei einem großen Teile der Richter und Staatsanwälte das Bedürfnis nach einer Patentlösung, die es ihnen ermöglicht, nach der Menge der illegal gehandelten Objekte zu entscheiden, ob Ordnungsstrafe, Wirtschaftsstrafbescheid oder gerichtliche Bestrafung am Platze ist - die es ihnen praktisch erspart, selbst darüber nachzudenken. Begründet wird dieses Bestreben mit der Befürchtung, daß seit dem Inkrafttreten der Verordnung zur Regelung des innerdeutschen Warenverkehrs ${ }^{3}$ eine ungleichmäßige Behandlung eintritt, da jetzt nicht mehr, wie bisher, die Entscheidung allein durch das Amt zur Kontrolle des Warenverkehrs ${ }^{4}$ erfolgt.

b) Weit verbreitet ist - vorwiegend bei den Richtern - die Auffassung, daß sie dem Angeklagten jedes Vorbringen restlos widerlegen müssen und daß sie, wenn ihnen das nicht

${ }^{1}$ Mit Rundverfügung vom 31.7. 1951, BAP, DP1 SE Nr. 3548, waren diese Länderkonferenzen angeordnet worden. Gesetz zum Schutze des innerdeutschen Handels (kurz: Handelsschutzgesetz) vom 21.4. 1950, in: Gesetzblatt der DDR 1950, S.327f.

2 Otto Grube (SED), Absolvent des 1. sächsischen Richterlehrgangs, tätig beim Generalstaatsanwalt der DDR.

${ }^{3}$ Verordnung zum Schutze des innerdeutschen Warenverkehrs vom 26.7. 1951, in: Gesetzblatt der DDR, 1951, S.705f. Während das Gesetz zum Schutze des innerdeutschen Handels sich nur auf den Warenverkehr zwischen West-Berlin und der DDR bezogen hatte, wurde mit der Verordnung vom 26.7. 1951 festgelegt, daß das Handelsschutzgesetz „für den gesamten Warenverkehr zwischen dem Gebiet der Deutschen Demokratischen Republik und dem übrigen Deutschland" gelten sollte.

4 In $\$ 2$ Handelsschutzgesetz war festgelegt, daß die Strafverfolgung , auf Antrag des Amtes für Kontrolle des Warenverkehrs" erfolgt; nach der Verordnung zum Schutze des innerdeutschen Warenverkehrs konnte entweder diese Dienststelle oder eine "Dienststelle der Wirtschaftsverwaltung" oder die Staatsanwaltschaft Strafverfolgung beantragen. Das Amt für Kontrolle des Warenverkehrs bestand seit dem 24.1. 1950. 
gelingt, keine Möglichkeit der Bestrafung aus dem Gesetz sehen. Diese falsch verstandenen Vorstellungen von der „Beweislast“ (die es im Strafprozeß überhaupt nicht gibt) spielen insbesondere bei den Behauptungen „Geschenke" und „Eigenverbrauch“ eine erhebliche Rolle.

c) Fast alle Richter und Staatsanwälte sehen bei der Bearbeitung in erster Linie den Angeklagten, der häufig ein Arbeiter oder Werktätiger ist, und nicht die Zusammenhänge und die Gefährdung der Wirtschaftsplanung. Daraus ergeben sich ihre Klagen, daß „das Volk dieses Gesetz und seine Maßnahmen nicht verstehe" und daß damit große Unzufriedenheit vorwiegend bei der werktätigen Bevölkerung angerichtet werde. Denn es handele sich oftmals um Menschen, die „sonst gute und mit unserer Entwicklung voll einverstandene Bürger" seien.

Verlangt wird eine weitgehende gründliche Popularisierung des Gesetzes, seiner Bedeutung und Folgen.

d) Oftmals weigern sich Schöffen, das Gesetz und seine Strafen anzuwenden. Infolge der hartnäckigen Weigerung sämtlicher Schöffen der Strafkammer Stendal (Vors[itzender] L[and]G[erichts]Präs[ident] Jüttner) mußte die Strafkammer nach stundenlanger Beratung wegen Vergehens gegen WStrVO ${ }^{5}$ zu einem Jahr Gefängnis verurteilen, obwohl einwandfrei der Tatbestand aus $\$ 2$, Abs. 2 des Gesetzes zum Schutze des innerdeutschen $\mathrm{Handels}^{6}$ vorlag und die Schöffen darauf hingewiesen worden sind, daß damit eine Rechtsbeugung begangen werde.

Aus diesen allgemeinen Eindrücken ist die Schlußfolgerung zu ziehen, daß es eine der wichtigsten Aufgaben sein muß, das ideologische Bewußtsein der Richter und Staatsanwälte zu heben. Offenbar ist die Einsicht in die politische Bedeutung der Gesetzgebung der Deutschen Demokratischen Republik im allgemeinen und des Gesetzes zum Schutze des innerdeutschen Handels im besonderen noch bei einem Teile der Richter und Staatsanwälte nicht vorhanden. Wenn sie ihre Aufgabe, unsere Wirtschaftsordnung zu schützen, voll anerkannt haben, sind sie in der Lage, selbst zu differenzieren, vermögen sie nach ihrer Lebenserfahrung zu beurteilen, was dem Angeklagten zu glauben ist, und muß es ihnen gelingen, sowohl [die] Bevölkerung als auch die Schöffen davon zu überzeugen, daß für den illegalen Handel eine harte Bestrafung am Platze ist.

\section{Besondere Diskussionspunkte in den einzelnen Veranstaltungen}

a) In Sachsen sind mehrfach Befürchtungen ausgesprochen worden, daß mit der Verordnung zum Schutze des innerdeutschen Warenverkehrs eine einheitliche Handhabung der Verfahren in Frage gestellt sei.

b) In Thüringen ist offenbar vom Referat eine Erleichterung in der Anwendung des Gesetzes dergestalt erwartet worden, daß den Richtern und Staatsanwälten Möglichkeiten zur Vermeidung der Härten aufgezeigt würden. Der erste Diskussionsredner (Kranke, Strafkammervorsitzender, Gera) schilderte in bewegten Worten die Lage einiger Angeklagten, bei denen es sich um tüchtige Arbeiter handeln soll, und erklärte, daß er es mit seinem Gewissen nicht vereinbaren könne, gerade diese Angeklagten nach dem Gesetz zu verurteilen. Er sei der Meinung, daß jedes Urteil, unter dem sein Name stehe, von seinem (scheinbar individuellen) Gewissen beantwortet [sic, wohl verantwortet] werden

\footnotetext{
${ }^{5}$ Verordnung über die Bestrafung von Verstößen gegen die Wirtschaftsordnung (Wirtschaftsstrafverordnung): siehe dazu Anm. 1 zu Dokument 33.

${ }^{6}$ In $\$ 2$, Abs. 2 des Handelsschutzgesetzes geht es um die „besonders schweren Fälle“, die mit „Zuchthaus nicht unter fünf Jahren und Vermögenseinziehung" bestraft werden sollen.
} 
müsse. Er führte weiter aus, daß die Wurzeln des Verbrechens in den gesellschaftlichen Verhältnissen liegen, und nach meinem Eindruck wollte er damit zum Ausdruck bringen, daß die gesellschaftlichen Verhältnisse in der Deutschen Demokratischen Republik noch manchmal zwingen, gegen die Gesetze zu verstoßen.

Der zweite Diskussionsredner (Knobs) fragte nach der Rechtmäßigkeit der Verordnung zur Regelung des innerdeutschen Warenverkehrs. Das Gesetz zum Schutze des innerdeutschen Handels sei von der Volkskammer erlassen, die Verordnung dagegen, die eine Erweiterung des Geltungsbereiches zur Folge habe, "nur" von der Regierung der Deutschen Demokratischen Republik. Nach seiner Meinung könne die Regierung nicht ein Gesetz der Volkskammer abändern.

Beide Fragen sind sehr deutlich und ausführlich vom Referenten beantwortet worden. Wie sich herausgestellt hat, sind beide Diskussionsredner Absolventen der Thüringer Richterlehrgänge und nach Meinung des Vertreters des Generalstaatsanwaltes im Lande Thüringen gute, politisch klare Staatsfunktionäre. Beide sind ehemalige Arbeiter, Kranke sogar aus der Maxhütte. Daraus kann nur die Schlußfolgerung gezogen werden, daß sie, die sich offenbar bei der Darbietung ihrer Diskussionsbeiträge sehr gefallen haben, durch die Richterschule und die Praxis einen wesentlichen Teil ihres Bewußtseins verloren haben.

c) In Sachsen-Anhalt ergab sich eine längere Diskussion über die Möglichkeit der "doppelten“ Bestrafung - Ordnungsstrafe durch das Amt zur Kontrolle des Warenverkehrs und gerichtliche Bestrafung. Ich habe den Eindruck, daß trotz der langen und ausführlichen Erläuterung eine volle Beseitigung aller Zweifel nicht erreicht worden ist.

III. Schlußfolgerungen

1. Die Durchführung einer Popularisierung der Vorschriften zum Schutze des innerdeutschen Handels und Zahlungsverkehrs ist notwendig, und zwar in

a) Justizausspracheabenden?

b) Prozessen vor erweiterter Öffentlichkeit,

c) Veröffentlichungen durch Presse ${ }^{8}$,

d) Aufklärung in größeren Betrieben durch Referate und evtl. auch Betriebsfunk,

e) Aufklärung durch Lautsprecher der Bahnhöfe bei Abfahrt von Zügen nach Berlin.

Bei allen Referaten, Prozessen, Veröffentlichungen und Ansagen ist das Schwergewicht auf die Schilderung des Schadens, der durch den illegalen Handel entsteht, zu legen. Richter und Staatsanwälte müssen in Plädoyer und Urteilsbegründung diesen Schaden ausführlich darlegen.

2. Besonderes Augenmerk ist auf die Schöffen in Wirtschaftsstrafsachen zu richten. Sie müssen über die Bedeutung des Gesetzes gründlich aufgeklärt werden. Sollten sie sich trotzdem weigern, das Gesetz anzuwenden, sind sie durch ihre Organisation zur Abberu-

${ }^{7}$ Dabei handelt es sich um Ausspracheabende über die Tätigkeit der Gerichte, bei denen die Richter und Staatsanwälte über ihre Tätigkeit Bericht erstatten sollten und die Bevölkerung sich informieren konnte. Diese Veranstaltungen dienten der Propagierung der justitiellen Praxis unter der Bevölkerung und der Rechtsberatung: siehe dazu die Runderlasse aus Mecklenburg und Thüringen vom Juli 1948, in: NJ 2 (1948), S. 161 f., und Rolf Helm, Öfentliche Justizveranstaltungen, in: Ebenda, S. $15 \mathrm{f}$.

${ }^{8}$ Für eine dieser Veröffentlichungen siehe Maximilian Stegmann und Otto Grube, Zu einigen Fragen bei der Anwendung des Gesetzes zum Schutze des innerdeutschen Handels, in: NJ 5 (1951), S.498500. 
fung aus dem Schöffenamt vorzuschlagen, sofern nicht noch weitere Maßnahmen notwendig sind.

3. Bei den Richtern und Staatsanwälten selbst ist die Hebung ihres politischen Bewußtseins eine der wichtigsten Aufgaben. Es fehlt bei einem großen Teil weniger am guten Willen, als an einer fundierten Überzeugung. Dazu ist eine gründliche Schulung, zunächst in Staatslehre, unbedingt notwendig, die etwa in folgender Weise durchzuführen ist:

a) Durchführung eines Lehrganges für die Lehrer und Seminarleiter im Ministerium der Justiz.

b) Durchführung von Lehrgängen in allen Ländern von etwa 4 Wochen Dauer, an denen alle Richter und Staatsanwälte, soweit sie nicht langfristige Lehrgänge an Partei- oder Verwaltungsschulen absolviert haben, teilnehmen müssen.

c) Nach Möglichkeit ist noch in diesem Jahre damit zu beginnen (Etat).

\section{Rundverfügungen, Schreiben und Berichte zum Einsatz der Volksrichter}

\section{Dokument 36}

Rundverfügung DJV an Landes- und Provinzialverwaltungen/Abteilung Justiz; Berlin, den 18.7. 1946

BAP, DP1 VA Nr.6335, Bl.111f., Konzept

Betrifft: Einsatz der aus den Lehrgängen für Richter und Staatsanwälte hervorgehenden Kräfte

In den Monaten August und September 1946 gehen die ersten Kurse für Richter und Staatsanwälte zu Ende. Mit Rücksicht darauf hat die SMAD in Berlin-Karlshorst ersucht, schon jetzt Vorkehrungen zu treffen, daß die aus den Kursen hervorgehenden Kräfte alsbald nach dem Bestehen der Abschlußprüfung in der am besten geeigneten Stelle als Richter oder Staatsanwälte eingesetzt werden ${ }^{1}$. Diese Aufgabe läßt sich am besten dadurch fördern, daß die Personalreferenten der Justizabteilungen sich unverzüglich durch Einholung von Beurteilungen seitens der Kursusleiter und -lehrer, nötigenfalls auch durch persönliche Besuche der Lehrgänge eine genaue Anschauung von den Fähigkeiten und Leistungen der Schüler verschaffen und auf Grund dieser Erkenntnisse die Verteilung der Absolventen auf die freien Stellen in den Justizbehörden vornehmen.

\footnotetext{
9 Gerda Grube, Absolventin des 1. sächsischen Richterlehrgangs, 1951 Hauptreferent im MdJ, nach der Flucht von Hans-Joachim Schoeps in den Westen im Dezember 1951 Leiterin der Abteilung Schulung.

1 Siehe dazu Vermerk Winkelmanns, 17.7. 1946, BAP, DP1 VA Nr.6335, Bl.111: „Bei dem Vortrag in Berlin-Karlshorst am 28.Juni 1946 ist sowohl vom Leiter der Rechtsabteilung, Karasjew, als auch von Oberstleutnant Lyssjak zum Ausdruck gebracht worden, schon jetzt müßten Vorkehrungen getroffen werden, um die aus den Lehrgängen für Richter und Staatsanwälte hervorgehenden Kräfte alsbald nach Bestehen der Abschlußprüfung zweckmäßig einzusetzen. Hierüber sollen die Länderund Provinzialverwaltungen durch eine Rundverfügung aufgeklärt werden."
} 
Nach den bisherigen Erfahrungen der Länder und Provinzen, die Richter und Staatsanwälte ohne Vorbildung beschäftigen, wird die Beachtung folgender Richtlinien empfohlen:

Am besten haben sich die neuen Kräfte in Staatsanwalts- und Amtsanwaltsstellen bewährt. Es empfiehlt sich daher, in erster Linie auch die Absolventen der Lehrgänge in diesen Stellen zu beschäftigen. Je nach ihrer Veranlagung dürften die aus den Kursen hervorgehenden Kräfte als Beisitzer in Strafkammern und Zivilkammern erster Instanz geeignet sein. Ihre Beschäftigung bei kleinen Amtsgerichten, an denen sie ohne die Möglichkeit einer Beratung als einzige Richter auf sämtlichen Rechtsgebieten tätig werden müssen, ist unbedingt zu vermeiden.

Darüber hinaus wird es erforderlich sein, eine Verschiebung innerhalb der augenblicklich besetzten Richter- und Staatsanwaltsstellen in der Weise vorzunehmen, daß mit dem Einsatz der Absolventen aus den Lehrgängen für Richter und Staatsanwälte Volljuristen an den Stellen eingesetzt werden, die für die neuen Richter bei ihrem Übergang in die Praxis noch nicht geeignet sind. Von der richtigen Verteilung des Einsatzes der neuen Richter wird es entscheidend abhängen, wie alle sich in der Rechtspraxis bewähren werden.

Ich erwarte daher, daß die vorbereitenden Maßnahmen in dem gekennzeichneten Sinne alsbald in Angriff genommen werden. Einem Bericht über das Veranlaßte sehe ich bis zum 1. August 1946 entgegen.

\section{Dokument 37}

Schreiben Ausbildungslehrgang für Richter und Staatsanwälte in Sachsen an DJV [gekürzt]; Bad Schandau, den 15.4. 1947 ${ }^{1}$

BAP, DP1 SE Nr.3561, Abschrift

Betrifft: Weitere Ausbildung der Teilnehmer am 1. Ausbildungslehrgang für Richter und Staatsanwälte

Aus den Berichten, die mir monatlich von den Teilnehmern selbst sowie von den Landgerichtspräsidenten bzw. Oberstaatsanwälten zugehen ${ }^{2}$, kann festgestellt werden, daß die eingesetzten Richter und Staatsanwälte bis auf zwei, und zwar die beiden Amtsrichterinnen Fräulein Doris Pilz in Leipzig und Frau Herta Minar in Freital, immer sicherer werden. Aus persönlichen Unterhaltungen mit einzelnen der Teilnehmer, mit denen ich wiederholt zusammenkomme, stelle ich fest, daß sie in dem neuen Beruf völlig aufgehen. Fast alle arbeiten zur Bewältigung der anfallenden Sachen bis spät in die Nacht hinein. Sie sind bestrebt, die vorliegenden, teilweise ganz erheblichen Reste so schnell als möglich aufzuarbeiten. Die Ursache für die lange Arbeitszeit ist nicht etwa die, daß sie zu viel Zeit für ihre Entschließungen benötigen, sondern die Anzahl der vorliegenden Akten. Einzelne der Teilnehmer sind bereits nicht nur als Straf-, Zivil- oder Vormundschaftsrichter tätig. Sie müssen vielmehr sämtliche Angelegenheiten eines Gerichts erledigen und finden sich in diesen mannigfachen Aufgaben bereits recht gut zurecht. In den meisten Fällen werden

\footnotetext{
${ }^{1}$ Das Schreiben wurde über das MdJ Sachsen an die DJV gesandt.

${ }^{2} \mathrm{Zu}$ dem Betreuungssystem für die Lehrgangsabsolventen im Land Sachsen siehe Einleitung, S. $82 \mathrm{f}$.
} 
von den beurteilenden Landgerichtspräsidenten und Oberstaatsanwälten der gute Vortrag und das gute Urteil hervorgehoben. In keinem Falle ist bisher etwas über langsames oder schwerfälliges Arbeiten berichtet worden. In einzelnen Fällen werden noch Mängel angezeigt in der Abfassung der schriftlichen Urteile, doch wird auch hier zu erkennen gegeben, daß diese Mängel mit der fortschreitenden Übung bald verschwinden werden. Die neun Besten sind vom Ministerium der Justiz bereits zu Amtsgerichtsräten ernannt wor$\mathrm{den}^{3}$, und zwar handelt es sich hierbei um

$[\ldots]^{4}$

Ein recht großer Teil [der] Teilnehmer hat in seinen Berichten mitgeteilt, daß die Schulungsbriefe ${ }^{5} \mathrm{Nr} .1$ und 4 bisher nicht eingegangen sind. Die Schulungsbriefe Nr. 2 und 5 dagegen, die von hier aus versandt worden sind, dürften jedem zugegangen sein, was daran liegt, daß die Anschriften immer auf dem Laufenden gehalten werden. Einzelne Teilnehmer haben nun gleichzeitig berichtet, daß es ihnen nicht möglich sei, die in den Schulungsbriefen gestellten Aufgaben zu bearbeiten, geschweige denn rechtzeitig der Deutschen Justizverwaltung zu übersenden, da sie derart mit beruflicher Arbeit überlastet sind, daß sie hierfür keine freie Zeit finden. Das ist auch der Grund, weswegen in Sachsen ein Vorschlag für die weitere Ausbildung der Lehrgangsteilnehmer durch bezirksweise Zusammenziehung noch nicht verwirklicht werden konnte. In der Zeit vom 5. bis 10. Mai 1947 findet in Bad Schandau für die Teilnehmer des 1. Lehrgangs die 2. Freizeit statt. Die Teilnehmer sind schon verständigt. An diesen Tagen wird das Wirtschaftssrafrecht anhand der von der Deutschen Justizverwaltung übersandten Zusammenstellung von Frau Dr. Koffk $a^{6}$ behandelt werden. Infolge Mangels von Matritzen konnte, was an sich beabsichtigt ist, noch nicht für jeden der Teilnehmer ein Exemplar angefertigt werden. Das Thema wird von $A[\mathrm{mts}] \mathrm{G}$ [erichts]Präsident Dr. Thust vorgetragen und im Seminar behandelt werden. Ein weiteres Thema über das Mietrecht hat Herr Ministerialrat Mannsfeld ${ }^{7}$ übernommen. Das dritte Thema über den Einfluß der neuen sächsischen Verfassung auf das bisher geltende Recht wird von dem Unterzeichneten behandelt werden. Dazu sind noch Einzelvorträge über Vernehmungstechnik, die Zusammenarbeit zwischen Richter, Staatsanwalt, Verteidiger, Verwaltung, Kommandantur der Roten Armee, Parteien, Gewerkschaften und Presse vorgesehen. Die endgültige Tagesordnung sowie ein Bericht über den Verlauf der Tagung werden der Deutschen Justizverwaltung zugesandt werden.

Der Lehrgangsleiter gez. Dr. Ebert

Ministerialrat

\footnotetext{
${ }^{3}$ Vorangegangen war ein Vorschlag Eberts: siehe Ebert an SED-Landesvorstand Sachsen, 19.2. 1947, BAP, DP1 SE Nr.3561.

${ }_{4}$ Ausgelassen wurde die Liste mit neun Namen und weitere Bemerkungen zu Personalfragen.

${ }^{5} \mathrm{Zu}$ den Schulungs- bzw. Unterrichtsbriefen siehe Anm. 19 zu Dokument 21.

${ }^{6}$ Dr. Else Koffka, Vortragender Rat in Abt VI. der DJV 1946-1948.

7 Zu Mannsfeld siehe Anm. 4 zu Dokument 32.
} 


\section{Dokument 38}

Bericht über die Dienstreise nach Thüringen vom 15. bis 17.Januar 1948 [gekürzt]; Berlin, den 24.1. 1948

$B A P, D P 1$ VA 1032, Bl.38-46, Original

I.

Der Zweck dieser Reise war, der Unterzeichneten die persönliche Kenntnis der im Lande Thüringen praktisch tätigen Absolventen des I. und II. Lehrgangs zu verschaffen. Zu diesem Zwecke wurden zunächst die Orte Gera, Erfurt und Weimar besucht, während der Besuch weiterer Gerichtsorte, in denen Absolventen der Kurse tätig sind, noch vorgesehen ist. Erreicht wurde dieses Ziel in Gera und Erfurt, während die drei in Weimar tätigen Staatsanwälte einer Aufforderung, sich um 1/2 2 Uhr auf dem Justizministerium einzufinden, nicht nachkamen, offenbar, weil sonnabends ihr Dienstschluß zeitiger liegt.

II.

Das allgemeine Bild der Stellung der neuen Richter und Staatsanwälte in Thüringen ist wie folgt zu kennzeichnen:

1) Es besteht der Eindruck, daß der Oberlandesgerichtspräsident Barth ${ }^{1}$, dessen Haltung bisher dafür sprach, daß er eine Aktivierung der Absolventen der Lehrgänge möglichst zurückzuhalten sucht, jetzt eine gewisse Wendung vorzunehmen scheint. Zwei Tage vor unserer Ankunft, am 13. und 14. Januar, hatte er in Gera eine Zusammenkunft aller in Thüringen tätigen Absolventen der Kurse zur Fortbildung einberufen, an der von insgesamt 30 Richtern und Staatsanwälten 25 teilgenommen haben. (Daß er es nicht für nötig gehalten hat, trotz des für den nächsten Tag vorgesehenen Besuches die Deutsche Justizverwaltung von dieser Veranstaltung zu informieren, ist charakteristisch für die thüringischen Beziehungen zur Deutschen Justizverwaltung. Immerhin war die Veranstaltung als solche positiv für die Richter und Staatsanwälte. Das Befremden über dies[es] Verhalten ist sowohl ihm wie Herrn Minister Külz ${ }^{2}$ gegenüber sehr offen zum Ausdruck gebracht [worden]).

Die Tagung hatte 2 Tage gedauert, und es waren ausschließlich praktische Fragen in seminaristischer Form behandelt worden. Sie hat bei den Teilnehmern, die von mir danach befragt wurden, Anklang gefunden, und es wurde dringend der Wunsch nach einer Wiederholung geäußert. Inzwischen ist vorgesehen, daß auf der Thüringer Verwaltungsschule in Finsterbergen, die der Justizverwaltung für 2 Wochen zur Verfügung gestellt wird, in nicht zu ferner Zeit ein zweiwöchiger Fortbildungskursus, auf dem dann auch zusammenhängende Vorträge gehalten werden sollen, abgehalten wird ${ }^{3}$. Herr Barth ist dringend ersucht, von dieser Veranstaltung der Deutschen Justizverwaltung rechtzeitig Mitteilung zu machen.

2) Die Stellung der Volksrichter, von denen ich in Gera 4 Richter und 4 Staatsanwälte und in Erfurt eine Richterin und 2 Staatsanwälte sprach, ergibt folgendes allgemeine Bild:

a) Es ist noch kein Absolvent der Kurse, auch nicht des I. Kursus, in eine Planstelle eingewiesen.

\footnotetext{
${ }^{1}$ Arno Barth (SPD/SED), 1945-1949 Präsident des thüringischen Oberlandesgerichts.

${ }^{2}$ Helmut Külz (LDP), 1946-1948 thüringischer Justizminister.

${ }^{3}$ Diese Fortbildungsveranstaltung fand vom 2.-14.8. 1948 statt; siehe den Bericht über diese Tagung in: BAP, DP1 VA Nr. 6335.
} 
b) Im übrigen unterscheidet sich die Stellung der neuen Kräfte bei der Staatsanwaltschaft wesentlich von der bei den Gerichten. Schon die Tatsache, daß von den insgesamt 23 Absolventen des II. Kursus 15 zur Staatsanwaltschaft und nur 8 zu den Gerichten gekommen waren, hatte den Schluß nahegelegt, daß man bei den Gerichten wesentlich weniger Wert auf die Tätigkeit der neuen Kräfte legt als bei den Staatsanwaltschaften. Dieses spiegelt sich auch in der Stellung im einzelnen wider.

Die Absolventen der Lehrgänge des I. Kursus haben nach 2 Monaten das kleine Zeichnungsrecht erhalten, das große ist ihnen für Februar in Aussicht gestellt ${ }^{4}$. Staatsanwalt Günther in Erfurt besitzt es als aufsichtsführender Staatsanwalt zur Durchführung des Befehls Nr. $201^{5}$ bereits jetzt. Zwei Staatsanwälte, Barckow und Scharf, sind abgeordnete Staatsanwälte in Sonneburg und Ilmenau in der Stellung eines Abteilungsleiters. Auch sie haben noch keine Planstelle.

Zwei von den neuen Richtern des Landgerichts Gera (Milkereit und Bloess) sind Absolventen des I. Lehrgangs. Was für ihre Stellung gilt, ist aber für alle bei den Gerichten tätigen Richter bestätigt worden. Sie werden ausschließlich als Beisitzer in Strafkammern und Berufungszivilkammern beschäftigt. Sie waren bisher weder als Amtsrichter bei größeren Amtsgerichten eingesetzt, noch sind sie zur Durchführung des Befehls Nr. 201 als Vorsitzende von kleinen Kammern herangezogen. In selbständiger Tätigkeit werden ihnen allein Ehesachen anvertraut. Dabei ergibt sich das befremdende Bild, daß - wohl wegen der juristischen Minderbewertung der Ehesachen - diese in einem Falle einem menschlich durchaus ungeeigneten, unausgeglichenen jungen Richter übertragen waren. Eine Ausnahme gilt weiter für den Absolventen des II. Lehrgangs Bunzel, der als einziger Richter auf das Amtsgericht Weißensee geschickt ist und um den der Landgerichtspräsident in Erfurt, wie er bestätigt, sich bisher noch nicht hat kümmern können. Die Stellung der Richter wird noch gekennzeichnet durch folgende Äußerung des Oberlandesgerichtspräsidenten Barth. Ich bedauerte, daß ich wegen der Kürze der Zeit - die Besprechung mit den Absolventen der Lehrgänge fand am Nachmittage des 15. Januar statt - nicht auch noch Gelegenheit hatte, mit den Vorsitzenden der Kammern zu sprechen, unter denen sie arbeiten. Herr Barth äußerte sich darauf etwa dahin, daß ich von diesen Herren auch nichts weiter erfahren würde.

Durch diese persönliche Beobachtung wird noch einmal bestätigt, was ja wahrscheinlich bereits aus anderen Anlässen geschlossen und hervorgehoben wurde, daß nämlich Thüringen es nicht verstanden hat und bisher vielleicht auch nicht hat verstehen wollen, die $\mathrm{Ab}$ solventen der Lehrgänge so bei den Gerichten einzusetzen, daß sie einmal als belebendes und fortschrittliches Element sich auswirken können und daß sie andererseits durch vielseitige Beanspruchung möglichst gut weitergebildet werden. Ich habe diese Beobachtung und meine daraus gezogenen Schlüsse Herrn Oberlandesgerichtspräsidenten Barth und Herrn Justizminister Külz sowie Herrn Oberstleutnant Schur ${ }^{6}$ ausführlich dargelegt. Herr Barth und Herr Külz schienen meine Ausführungen anzuerkennen und stellten eine Änderung des Einsatzes der neuen Richter in Aussicht. Bemerkt sei noch, daß Herr Justiz-

\footnotetext{
4 Auf Nachfrage von Oberstleutnant Jakupow wurden die Begriffe in einem Schreiben an die SMADRechtsabteilung am 2.3. 1948 wie folgt erläutert: „Unter, großem Zeichnungsrecht ist die volle selbständige Unterschriftsbefugnis, insbesondere unter Anklage- und Einstellungsbeschlüssen, zu sehen, während das ,kleine Zeichnungsrecht" nur für weniger wichtige Verfügungen und Anordnungen gegeben ist." BAP, DP1 VA Nr. 6335, Bl. 164.

5 Siehe dazu Einleitung, S. 87.

6 Oberstleutnant Schur, Abteilung Justiz bei der Sowjetischen Militäradministration in Thüringen.
} 
minister Külz überrascht schien, daß überhaupt noch keiner der neuen Richter in eine Planstelle eingewiesen ist.

III.

$[\ldots]^{7}$

Es wurden mit sämtlichen Richtern in längeren Aussprachen sowohl die Stellung der Volksrichter im allgemeinen erörtert wie auch Fragen persönlicher und sachlicher Art beantwortet. Neben dem schon oben erwähnten Wunsch auf baldige Veranstaltung eines längeren Fortbildungskurses ist noch hervorzuheben, daß sie besonders den Wunsch äußerten, näheres über das Schicksal der von ihnen verfaßten Urteile in der Revisionsinstanz zu erfahren. Es wurde ihnen empfohlen, sich regelmäßig bei der Geschäftsstelle zu erkundigen, ob die von ihnen verfaßten Urteile bereits wieder zurückgegangen sind, und darüber hinaus anzuregen, daß ihnen solche Urteile vorgelegt werden. Sie regten weiter an, daß es zu einer regelmäßigen Besprechung typischer Fehler ihrer Urteile, insbesondere auf Grund der Erfahrungen in der Revision kommen möge.

An materiellen Wünschen wurde die bekannte Klage erhoben, daß sie immer noch nicht die Lebensmittelkarte $\mathrm{II}^{8}$ haben. Die jüngeren Richter führten darüber Klage, daß sie auf Grund der Besoldungsbestimmungen erst vom 28. Lebensjahre an mit einer Steigerung ihres Gehaltes rechnen können. Ich habe ihnen dazu gesagt, daß sie einmal eigentlich überhaupt zu jung wären, um Richter zu sein und daß im übrigen die Fragen der Besoldung im Fluß seien.

Zusammenfassend ist festzustellen, daß der Eindruck dieser Richter durchaus positiv ist. Es ist jedoch notwendig, sie nunmehr wesentlich beweglicher einzusetzen, ihr Können in größerem Umfange nutzbar zu machen (es sei nur darauf hingewiesen, daß seit dem 1.12. 1947 in Thüringen 8 Richter gestorben sind und 6 auf Monate hinaus erkrankt sind), um entstehende Lücken auszufüllen.

$[\ldots]^{9}$

Benjamin

\section{Dokument 39}

Rundverfügung Chef der DJV an Landesregierungen/Justizministerium [Auszug]; Berlin, den 20.9. 1948

\section{BAP, DP1 VA Nr.6335, Bl.171-177, Durchschrift}

Betrifft: Juristentagungen

Am 11. und 12.Juni 1948 hat bei der Deutschen Justizverwaltung in Berlin die Juristenkonferenz stattgefunden ${ }^{1}$, der sich entsprechende Konferenzen in allen Ländern der sowjetischen Besatzungszone angeschlossen haben und die sich von den bisherigen Länder-

${ }^{7}$ Ausgelassen wurden die Berichte über 11 in Thüringen eingesetzte Lehrgangsabsolventen.

8 Siehe Anm. 3 zu Dokument 18.

9 Ausgelassen wurde der letzte Absatz, in dem Benjamin über ein Gespräch mit Külz berichtet. Darin ging es um die Möglichkeit, Personen ohne Vorbildung als "Hilfsarbeiter" bei den Staatsanwaltschaften einzusetzen, um die Durchführung von Befehl Nr. 201 und um Personalangelegenheiten.

${ }^{1}$ Siehe Einleitung, S. 49. 
konferenzen dadurch grundsätzlich unterschieden hat, daß auf ihr nicht einzelne juristische Spezialprobleme behandelt, sondern die für die praktische Arbeit der Justiz bedeutsamsten grundsätzlichen Fragen einer Erörterung unterzogen wurden. Durch die Ausgestaltung der verschiedenen Konferenzen ist erreicht worden, daß praktisch alle Angehörigen der Justiz der sowjetischen Besatzungszone die Referate, die sich mit den Hauptaufgaben der Justiz befaßten, gehört haben. Die Deutsche Justizverwaltung hat außerdem ein Sonderheft der „Neuen Justiz“ herausgegeben, um den Richtern und Staatsanwälten sowie allen anderen Angehörigen der Justiz die Möglichkeit zu geben, sich an Hand der in diesem Sonderheft veröffentlichten Referate eingehend mit den in diesen aufgeworfenen Fragen und Problemen zu beschäftigen².

Als Ergebnis der Konferenz sind in den einzelnen Ländern Resolutionen angenommen worden ${ }^{3}$, die dem Ausdruck gegeben haben, was als Ergebnis der Diskussionen und der gemeinschaftlichen Arbeit dieser Konferenzen festgehalten zu werden verdient. Wenn aber das mit den Konferenzen verfolgte Ziel, nämlich die weitere Demokratisierung der Justiz in der sowjetischen Besatzungszone, erreicht werden soll, so genügt es nicht, Referate zu halten oder anzuhören und Resolutionen zu fassen. Dieses Ziel kann vielmehr nur erreicht werden, wenn in der praktischen Arbeit der Justiz das verwirklicht wird, was in den Referaten und in den Resolutionen als Forderung aufgestellt worden ist.

Die Deutsche Justizverwaltung beabsichtigt, im Oktober 1948 eine zweite Konferenz dieser Art zu veranstalten ${ }^{4}$. Auf dieser Konferenz wird vor den Vertretern der Justiz der einzelnen Länder der Zone die Aufgabe stehen, Bericht zu erstatten über das, was zur Durchführung und zur Verwirklichung der auf den Konferenzen entwickelten Grundsätze in ihren Ländern geschehen ist. Dieser Rechenschaftsbericht wird sich, entsprechend den Themen, die auf den Konferenzen behandelt worden sind, auf folgende Fragen beziehen müssen:

I. In dem grundlegenden allgemeinen Referat ${ }^{5}$ hat Herr Fechner die Linie aufgezeigt, auf der sich nach dem Abschluß der ersten Periode der Demokratisierung der Justiz der sowjetischen Besatzungszone, die gekennzeichnet ist durch die Bereinigung des Personalbestandes von nazistischen Elementen und durch die Beseitigung des alten zweifellos nicht mehr anwendbaren Rechtes, die weitere Demokratisierung der Justiz in unserer Zone zu bewegen hat. In der Weimarer Resolution ${ }^{6}$, die im Anschluß an die thüringische Juristenkonferenz von den Teilnehmern aller folgenden Konferenzen einstimmig angenommen worden ist, sind die wesentlichsten Prinzipien für diese Arbeit zur weiteren Demokratisierung der Justiz zusammengestellt worden.

1) Hiernach ist in erster Linie entscheidendes Gewicht auf die Hebung des fachlichen und politischen Niveaus der Angehörigen der Justiz zu legen. Dieser Arbeit kommt deshalb eine so entscheidende Bedeutung zu, weil nur durch den Einsatz und die Tätigkeit fachlich und politisch qualifizierter Menschen das Ziel der weiteren Demokratisierung der Justiz erreicht werden kann. Die Vertreter der Länder mögen deshalb auf der kommenden zweiten Juristenkonferenz darüber berichten, was geschehen ist,

\footnotetext{
${ }^{2}$ Siehe: Die Juristenkonferenzen in der sowjetischen Besatzungszone Deutschlands - Juni-August 1948, in: NJ 2 (1948), S. 121-140.

${ }^{3}$ Ebenda, S. 139 f.

4 Diese Konferenz fand erst am 25./26.11. 1948 statt.

5 Das Referat trägt den Titel: „Aufgaben der weiteren Demokratisierung der Justiz“, in: NJ 2 (1948), S. 121-126.

- Ebenda, S. $139 f$.
} 
a) um die Richter, Staatsanwälte, aber auch die Rechtsanwälte mit den allgemeinen wirtschaftlichen und politischen Problemen vertraut zu machen, die im Vordergrund ihrer heutigen Arbeit stehen (Zweijahresplan, Volkseigentum, Bodenreform etc.);

b) um die Angehörigen der Justiz durch laufende Fortbildungs- und Ausbildungskurse wie auch durch besondere Veranstaltungen auf fachlichem Gebiete weiter zu bilden;

c) um die Arbeit an den Richterschulen zu verbessern, insbesondere dadurch, daß eine sorgfältigere Auswahl der Lehrkräfte als bisher vorgenommen und auch den Lehrkräften selbst die Gelegenheit gegeben wird, sich weiter zu bilden.

2) Die Personalpolitik in der Justiz der Zone muß von dem Grundsatz geleitet sein, daß nur solche Richter und Staatsanwälte für die weitere Arbeit in der Zone tragbar sind, die die demokratischen Errungenschaften, die in der sowjetischen Besatzungszone erreicht worden sind, bejahen und sich zu ihnen bekennen. Die Arbeit der Richter und Staatsanwälte auf den Gebieten, die im Vordergrund des allgemeinen Interesses stehen, bei der Bekämpfung der Naziverbrecher, der Saboteure des Wirtschaftsplanes, der Schädiger des Volkseigentums, der Bauern, die ihre Ablieferungspflicht verletzen, muß Maßstab dafür werden, wer bei der Beförderung und bei der Neubesetzung von Stellen in erster Linie zu berücksichtigen ist. Dabei kann es keinen Zweifel mehr darüber geben, daß bei den Fragen der Neubesetzung von Stellen und der Beförderung ein Unterschied zwischen den Absolventen der Richterkurse und den akademisch vorgebildeten Richtern nicht mehr gemacht werden kann. Die Absolventen der ersten Richterlehrgänge haben genug Erfahrungen gesammelt, um als Aufsichtsrichter, als Vorsitzende von Kammern der Landgerichte, und zwar auch von Berufungskammern, sowie als Oberstaatsanwälte eingesetzt zu werden. Ihre Tätigkeit im Rahmen der Durchführung des Befehls 2017 hat bewiesen, daß sie in der Lage sind, diese Stellen voll auszufüllen. Gerade die Richter und Staatsanwälte aber, die bisher in den Verfahren nach dem Befehl 201 tätig gewesen sind, sollten nach und nach in die gewöhnliche Praxis zurückgeführt werden ${ }^{8}$.

Mit allem Nachdruck ist darauf hinzuweisen, daß die Arbeit für die Auswahl der Anwärter für die Richterschulen verbessert werden muß. Über die Erfolge gerade dieser Arbeit zu berichten, wird eine der wesentlichsten Aufgaben der Vertreter der Länder bei ihren Rechenschaftsberichten auf der nächsten Konferenz sein.

$$
[\ldots]^{9}
$$

In Vertretung

Dr. Melsheimer

\footnotetext{
${ }^{7}$ Siehe Einleitung, S. 87.

${ }^{8}$ Dieser Abschnitt wurde weitgehend nach den Vorgaben von Hilde Benjamin formuliert: siehe Benjamin an Weiß, 16.9. 1948, BAP, DP1 VA Nr.6335, Bl.170.

9 Die weiteren Abschnitte betreffen weder den Einsatz noch die Fortbildung der Volksrichter.
} 


\section{Dokument 40}

Rundverfügung Chef der DJV an Landesregierungen/Justizministerium; Berlin, den 23.12. 1948

BAP, DP1 VA 7844, Bl. 298, Durchschrift

Betrifft: Entschließung der Juristenkonferenz vom 26. November 1948 - Beförderung von Absolventen der Richterlehrgänge ${ }^{1}$

Mit meiner Rundverfügung vom 22. Oktober 1948 - $2200 \mathrm{Ec} / 3$ - II 1259/48 - ${ }^{2}$ hatte ich Bericht darüber erbeten, in welchem Umfange die Beschlüsse der Juristenkonferenz vom Sommer diesen Jahres, Absolventen der Lehrgänge in gehobene Stellungen zu befördern, bis zum 1.November ausgeführt worden waren.

Sowohl die schriftlichen Berichte wie auch die Mitteilungen der Justizminister auf der Konferenz am 25./26. November haben noch kein befriedigendes Ergebnis gezeigt. Es muß vielmehr festgestellt werden:

1) die Beförderungen beschränken sich zu einem großen Teil (fast zu 50\% aller Beförderungen) auf die Bestellung zum Aufsichtsrichter;

2) die Tätigkeit der Vorsitzenden von Berufungskammern wird den Absolventen nach wie vor vorenthalten;

3) soweit man Absolventen in einer gehobenen Stellung tätig sein läßt, hat man sie sehr häufig nicht in die entsprechenden Stellen eingewiesen. Das gilt insbesondere für die Vorsitzenden der Kammern der Landgerichte, die nicht zu Direktoren befördert wurden.

Die Personallage in der Justiz aller Länder der SBZ verlangt insbesondere auch bezüglich der Besetzung der Stellen der Landgerichtspräsidenten und Landgerichtsdirektoren gebieterisch, daß auf die Kräfte aus den Lehrgängen zurückgegriffen wird.

Dabei lenke ich in diesem Zusammenhang die Aufmerksamkeit auch auf den Punkt der Entschließung vom 26.11. 1948, daß alle Fortbildungsmöglichkeiten, insbesondere auch zonale wie die Deutsche Verwaltungs-Akademie in Forst Zinna, auszuschöpfen sind ${ }^{3}$. Diese Lehrgänge sind ganz besonders geeignet, um die Teilnehmer für gehobene Stellungen vorzubereiten.

Ich erbitte Bericht über die im Sinne dieser Verfügung seit dem 1. November 1948 getroffenen Maßnahmen bis zum 1. Februar 1949, wobei ich entsprechend der Rundverfügung vom 22.10. $1948 \mathrm{zu}$ verfahren bitte.

Fechner

${ }^{1}$ Die einschlägige Entschließung lautet: „Die Absolventen der Lehrgänge sind, obwohl das in der Weimarer Entschließung gefordert worden war, bei der Besetzung verantwortlicher Stellen noch nicht in dem Maße gefördert worden, wie es ihrer Bedeutung für die Demokratisierung der Justiz entspricht", in: NJ 2 (1948), S. 266.

2 DJV an Landesregierungen/Justizministerium, 22.10. 1948, BAP, DP1 VA Nr.6335, Bl.188; siehe dazu Einleitung, S. 90.

${ }^{3}$ Der einschlägige Teil der Entschließung lautet: „Bei dieser Weiterbildung sind alle Fortbildungsmöglichkeiten, auch die zonalen wie z. B. die Deutsche Verwaltungsakademie in Zinna, auszuschöpfen", in: NJ 2 (1948), S. 266. Zu den Qualifizierungskursen für Juristen bei der DVA siehe Einleitung, S. 78 . 
